\title{
The Relationship Between Surface Wear and Contact Resistance During the Fretting of In-Vivo Electrical Contacts
}

\author{
John W. McBride
}

\begin{abstract}
A study of the high frequency intermittency events occurring during the fretting of contact surfaces used for in-vivo electronic systems, is presented. The emphasis of the study is to determine and the relationship between the contact resistance during the fretting process and to relate this to surface wear, as a function of the applied force. The emphasis is on fretting experiments in the crossed rod configuration with a range of contact forces, between 1.75 and $0.05 \mathrm{~N}$.

An established test system and test methodology are used for the study, but in the paper the additional control of the contact force and the measurement of the surface wear using a 3-D surface scanning system is introduced. The level of the applied voltage and current are critical in defining the intermittency events; in this study a dry circuit test, with $20 \mathrm{mV}$ and $100 \mathrm{~mA}$ supply is used. The results show that for the materials used, intermittency events occur during every fretting cycle after an initial settling period; that the contact force level is directly coupled to the wear in-line with established theory; and that the minimum contact resistance is linked to the established relationship with force.
\end{abstract}

Index Terms-Connectors, fretting, in-body electronics, in-vivo systems, intermittency, surface wear.

\section{INTRODUCTION}

$\mathbf{T}$ HIS paper presents a study of the electrical contact performance of bio-compatible electrical contact surfaces. In particular the focus is on the intermittency performance of these surfaces under low frequency fretting conditions aimed to replicate the types of motion that may be experienced in-vivo. Fretting motion is generally cyclic with amplitude and frequency depending on the driving mechanism. The amplitude of fretting is generally in the range of a few micrometers to a few hundred micrometers. The contact surfaces selected are used primarily in the application of in-body or in-vivo electronic systems, and the study presented here follows on from an initial investigation into this application [1]. Fretting is known to be a major cause of contact deterioration and failure; commonly exhibited as the contact resistance increases from a few milliohms $(\mathrm{m} \Omega)$, in the case of a new contact, to in excess of several ohms. In addition to the gradual increase in contact resistance, intermittently high contact resistance can occur. These events occur in electronic

Manuscript received December 4, 2006; revised March 28, 2008. Current version published September 17, 2008. This work was recommended for publication by Associate Editor T. Schoepf upon evaluation of the reviewers comments.

The author is with the School of Engineering Sciences, University of Southampton, Southampton SO17 1BJ , U.K., (e-mail: jwm@ soton.ac.uk).

Color versions of one or more of the figures in this paper are available online at http://ieeexplore.ieee.org.

Digital Object Identifier 10.1109/TCAPT.2008.2001162 connector systems where the conduction path across the electrical contact interface is disrupted for a short duration [2]-[5]. These events can cause difficulties with electronic systems and result in system failure. Although the occurrence of intermittencies has been known for a number of years, the phenomenon is generally overlooked in traditional fretting experiments, where the instruments normally used to measure contact resistance are unable to record the rapid changes in contact resistance. [6]. Intermittency has been shown to result from the loss of metallic conduction between contact surfaces, [4], and is therefore generated by the interactions with oxides and other surface contaminants as the two surfaces move. The events considered can be of the order of a few $\mu$ s to a few milliseconds, and are detected using a dynamic threshold method. In [1] a model was proposed to explain the wear process however the model does not account for contact force variation. The model is reconsidered in this study, and includes the influence of contact force.

\section{A. Initial Model For the Intermittency Mechanism in Crossed-Rod Contact Pairs}

The following mechanism was proposed as the process by which the intermittency events evolve, in "crossed rod" contacts with fretting motion. For the crossed rod geometry the rider is the contact with the smaller of the two cylindrical cross sections. A three stage process was proposed.

1) As the contacts are mated the act of assembly is expected to damage the oxide surface layer, as the fretting motion starts the contact will move to new areas covered in oxide. Thus the initial stage of the fretting is expected to show a high number of intermittent events. The contact geometry at this stage will be a point contact on the rider, acting on a fretting line on the coupon, shown in Fig. 1(a).

2) As the contact surface wears the point contact on the rider will change such that the apparent surface area increases, as shown in Fig. 1(b). This stage is considered to be the period of stability where the oxides are removed, resulting in a minimization of intermittency.

3) As the fretting cycles increase more surface wear occurs on the rider and the wear track on the coupon will expand in a direction perpendicular to the motion, A, in Fig. 1(c). As this occurs more of the surface oxide interactions will occur toward the edges of the wear track. The number of intermittency events will increase. This will continue as the samples continue to wear.

The proposed model was based on the observed results from connector surfaces with no control of the contact force. In this 

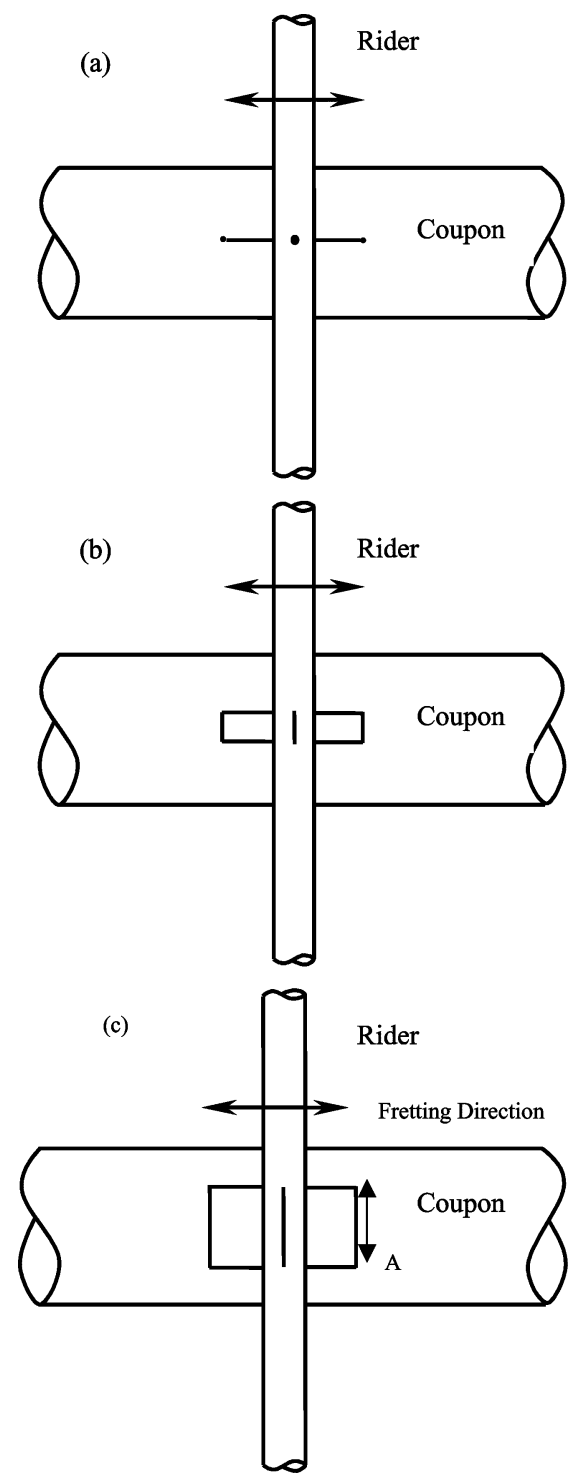

Fig. 1. Schematic of the proposed three-stage wear process using a cross-rod configuration, with the coupon surface corresponding to the larger cylinder surfaces, and the rider the smaller cylinder.

study the test procedure has been constrained to investigate the underlying phenomena. A crossed-rod configuration is used, where the contact force is controlled, and the surface wear investigated.

To enable the relationship with surface wear the Archard equation for adhesive wear can be used; where the volumetric wear $(V)$ is proportional to the load $(F)$ and the distance travelled $(L)$

$$
V=\frac{K F L}{3 H}
$$

where $K=$ wear coefficient; and $H=$ hardness;

For a fretting experiment, where $N$ is the total number of cycles, and $x$ is the fretting displacement

$$
V=\frac{2 K F N x}{3 H} \text {. }
$$

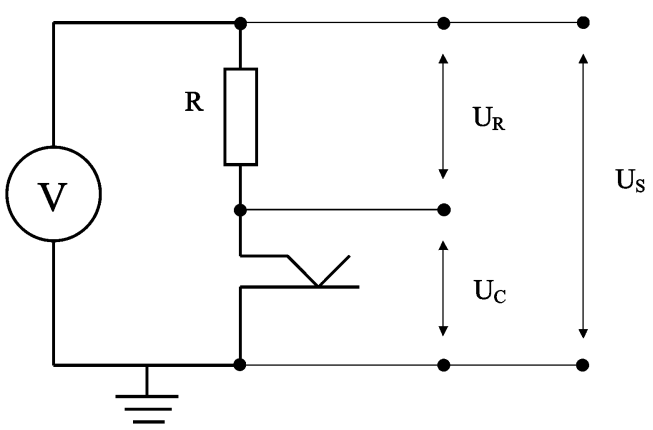

Fig. 2. Contact voltage-drop and contact current measuring circuit.

\section{EXPERIMENTAL METHODS}

A test methodology is presented based on a previous study where connector surfaces were used [1]; in the current study crossed-rod contacts are used with control of the contact force.

\section{A. Overview}

A high frequency intermittency testing station has been used for the study [1], [2], [4]. It consists of a motion system that moves one contact member (the coupon) in the horizontal plane to create the fretting motion while maintaining the contact force of the second contact (rider). The vertical axis is used to control contact force. This creates a system where the contact force is held constant through the fretting and wear processes. This does not recreate a real contact surface but allows the control of a important parameter for the wear study. The system is mounted on a vibration isolation workstation in a temperature controlled clean room. $\left(20^{\circ} \mathrm{C}\right)$. A $20 \mathrm{mV}$ power supply is used creating dry a circuit condition. The custom built power supply delivers a maximum of $100 \mathrm{~mA}$ through a current limiting resistor, shown in Fig. 2.

\section{B. Contact Terminals}

The material selected for this study is a solid nickel-cobalt-chromium-molybdenum alloy possessing a unique combination of high tensile strength, good ductility and toughness, and excellent corrosion resistance. A full discussion of contact materials for in-vivo applications is reported in [1]. The alloy melting temperature is $1315{ }^{\circ} \mathrm{C}-1440{ }^{\circ} \mathrm{C}$, the corresponding contact voltage drop required to cause surface melting is $0.51 \mathrm{~V}$. The contacts are mounted in a crossed rod configuration with one element having a radius of $r_{1}=1.63 \mathrm{~mm}$, (referred to here as the coupon) with the other sample with radius $r_{2}=0.163 \mathrm{~mm}$ (referred to as the rider) as shown in Fig. 1. The (10:1) difference in radius is selected to enhance the wear profile between the samples. The selection of a solid alloy surface is important, as the majority of fretting studies are conducted with plated surfaces, where the interaction with the base surfaces due to plating wear leads to added complexity in understanding the underlying physical processes.

\section{Fretting Cycle}

The fretting displacement used is $100 \mu \mathrm{m}$. The stages accelerate within $1.0 \mathrm{~s}$, remain at a constant velocity for $1.0 \mathrm{~s}$ and 
decelerate to a halt within another $1.0 \mathrm{~s}$. The total cycle time is $8 \mathrm{~s}$.

\section{Contact Voltage and Current}

The contact deterioration is measured in terms of the contact volt-drop, since the condition of the contact interface is characterized by the asperity temperature and therefore the voltagedrop across the contact interface. Fig. 2 shows the circuit used for the study where $V=20 \mathrm{mV}$. Particular attention has been paid to keeping the signal path for current and voltage as simple as possible, as each additional amplifier would decrease the time response of the overall system. The contact voltage-drop $U_{C}$ and the supply voltage $U_{\mathrm{S}}$ are measured with a high-speed digital storage oscilloscope, capable of sampling two channels at a rate of $1 \mathrm{~Gb} / \mathrm{s}$ each with a vertical resolution of 8 bit. The voltage-drop across the resistor $\mathrm{R}$ is calculated by subtracting the contact voltage-drop. The relationship between the contact voltage $\left(U_{c}\right)$ and the contact resistance is non-linear. A model for this has been presented previously [1], and is used here to convert data to contact resistance values used in the results.

\section{E. Test Procedure}

The samples are cleaned and mounted in the test fixture. Each sample pair is then mated to the required contact force. Each sample pair is tested for 2500 cycles. ( $\sim 24$ hours $)$. Four contact force levels are used, $1750 \mathrm{mN}, 750 \mathrm{mN}, 150 \mathrm{mN}$, and $50 \mathrm{mN}$. A methodology was established in a previous study for the evaluation of the intermittency events with a low level power supply voltage, [1]. In summary, an event is recorded if there is a change in the contact voltage over the $1 / 2$ cycle. The method used to define the intermittency event with a $20-\mathrm{mV}$ power supply is as follows; it should be noted that this method differs from that used with a 5-V power supply [1].

1) The data acquisition is triggered at the start of each half cycle.

2) The voltage across the contact is processed to determine the average voltage, the standard deviation of the whole waveform and the average voltage each 1/20th of the waveform.

3) An intermittent event is logged when any of the $1 / 20$ th average values is greater than the mean based on the following criteria:

$$
\overline{U_{C}}\left(\frac{1}{20}\right)>\overline{U_{C}} \pm 2 \sigma .
$$

4) If the criteria is not met then no data is recorded.

\section{F. Wear Analysis}

The surface wear is analysed using the TaiCaan Technologies XYRIS4000CL precision 3-D surface scanner. The system provided 3-D surface evaluation with 10-nm resolution in all axes, and is supported by software which performs the surface volumetric wear calculations on surfaces with surface form, for example the cylindrical surfaces used in this study [7], [8]. In the method used the wear area is removed prior to the form fitting process, the volumetric wear is then more accurately defined.

\section{RESULTS}

\section{A. Intermittency and Surface Wear At A Contact Force of $1750 \mathrm{mN}$}

Fig. 3 shows the number of intermittency events recorded every 20 cycles. With an event recorded every half cycle the maximum number of event is 40 . It is observed that over most of the 2500 cycles there are events every half cycle. The data presented is a direct result of the wear process highlighted in the introduction. In Fig. 3, regions (A to D) of intermittency are identified, which are related to the 3 stage model previously discussed. In Fig. 4 the Mean, Maximum and Minimum contact resistance are shown, over 100 and 2500 fretting cycles. Referring to the 4 regions in Fig. 3 and to Fig. 4 :

A) Corresponding to stage 1 . The initial period of events as the oxide is removed from the surface. This is characterized with an increase in the contact resistance value. Using the mean value, the resistance increases from $0.12 \Omega$, to $0.15 \Omega$, and then reduces as the oxide is worn away to approximately $0.11 \Omega$, over the first 100 cycles. (Two values are presented for each of the cycles corresponding to the mean resistance for each half cycle)

B) Corresponding to stage 2, occurring over 200 to 500 cycles. During this phase only a few intermittency events occur. This corresponds to a period where the oxide has been removed and where the conductance is nearly $100 \%$ metallic. With only a limited number of intermittency events recorded the mean contact resistance shows a stable level of approximately $0.08 \Omega$.

C) Corresponding to stage 3 . In the third section the rider and coupon interact with the oxides pushed to the edges of the wear area, leading to an increase in intermittency. The corresponding mean contact resistance shows an increase from $0.08 \Omega$ to a peak at 700 cycles of $0.16 \Omega$ before reducing to a stable level of $0.07 \Omega$, at 1300 cycles.

D) The process exhibits a new stage, where every $1 / 2$ cycle is associated with an event. The corresponding mean contact resistance data shows a stable resistance of approximately $0.07 \Omega$.

The results show an increased complexity to the wear process, with an apparent 4 stage process. To investigate the relationship between the intermittency events and the surface wear both the rider and the coupon have been investigated for 3 -D volumetric wear, after 2500 cycles.

1) Coupon Surface Wear

Fig. 5 shows the resultant coupon wear after 2500 cycles, the form of the surface has been removed using a second order polynomial, allowing wear values to be determined relative to the flat datum surface. The wear data is shown in Table I, for all force levels. For the $1.75 \mathrm{~N}$ force the overall length of the wear track, $260 \mu \mathrm{m}$, includes the wear debris field. The inner wear scar length of $100 \mu \mathrm{m}$ corresponds to region below the nominal surface in the $2-\mathrm{D}$ cross section, and to the fretting amplitude. The 2-D section across the wear area shows surface removed to a depth of $10 \mu \mathrm{m}$.

2) Rider Surface Wear 


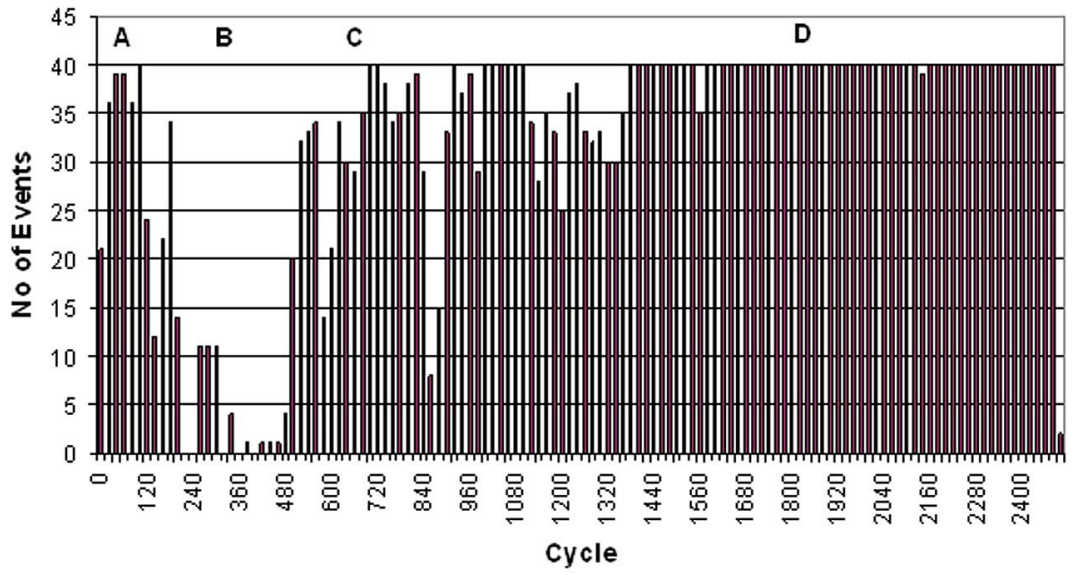

Fig. 3. Number of intermittency events per 20 cycles over 2500 cycles during intermittency events at a constant contact force of $1750 \mathrm{mN}$.

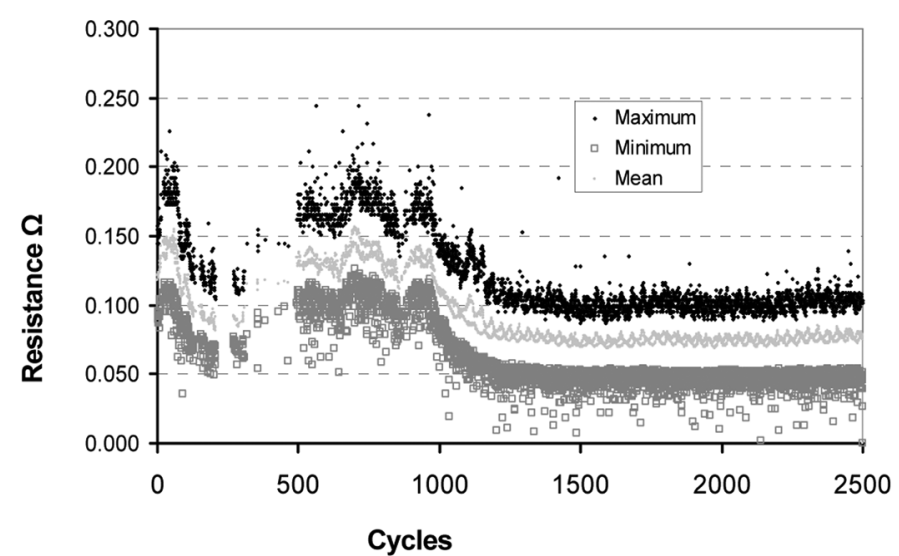

(a)

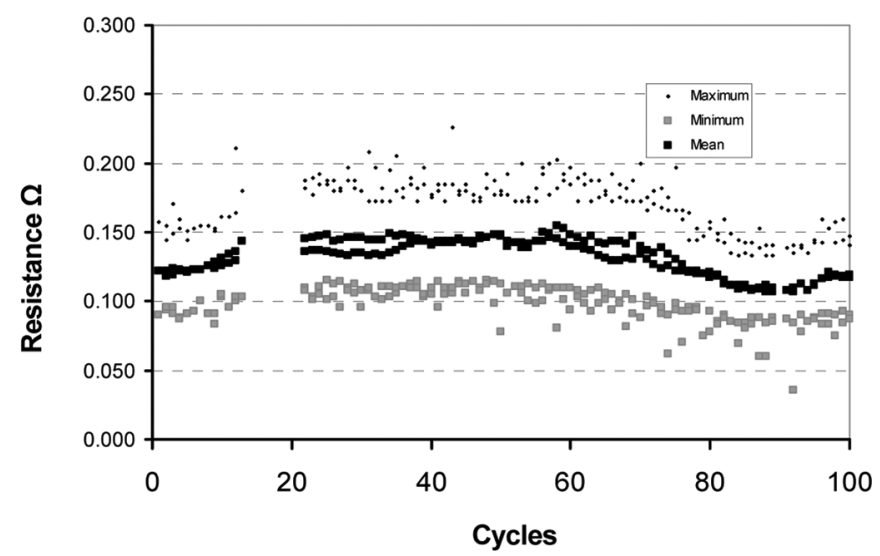

(b)

Fig. 4. Mean, max, and min contact resistance over (a) 2500 and (b) 100 cycles during intermittency events at a constant contact force of $1750 \mathrm{mN}$.

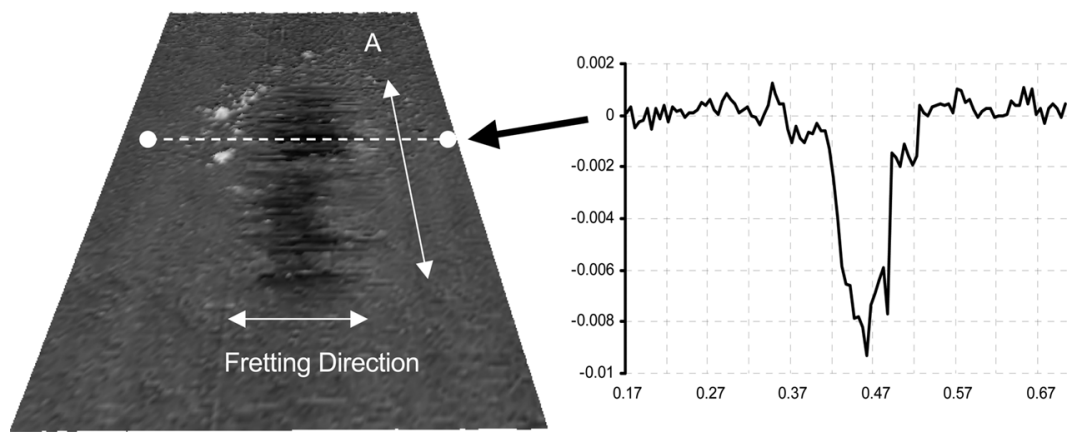

Fig. 5. Surface wear of coupon with the cylinder form is removed. (left) Showing the fretting direction of the rider, A is the length of the wear area and (right) the 2-D cross section, all dimensions ( $\mathrm{mm}$ ).

Fig. 6 shows the rider surface mated to the coupon surface in Fig. 5. The rider surface becomes curved by the wear process, reflecting the radius of the coupon surface. A circle fit identifies the radius of the removed material to be $1.627 \mathrm{~mm}$. The resultant wear data is shown in Table II. The wear analysis shows that the majority of the volumetric wear occurs on the rider, with $31.5 \times 10^{-5} \mathrm{~mm}^{3}$ of material lost, compared to $8.8 \times 10^{-5} \mathrm{~mm}^{3}$ material lost from the coupon surface. The lost material is assumed to fall free from the surface.

\section{B. Intermittency and Surface Wear At Contact Force of $750 \mathrm{mN}$}

The contact resistance data is shown in Fig. 7. The data over the first 100 cycles shows the contact resistance increasing over the first few cycles to $0.6 \Omega$ then falling at around 10 cycles (to $0.2 \Omega$ ), then increasing again to peak at 40 cycles $(0.4 \Omega)$, before falling again to the $0.2 \Omega$ level exhibited over most of the response in Fig. 7 over 2500 cycles. Towards the end of the period after 1500 cycles the mean contact resistance characteristic 


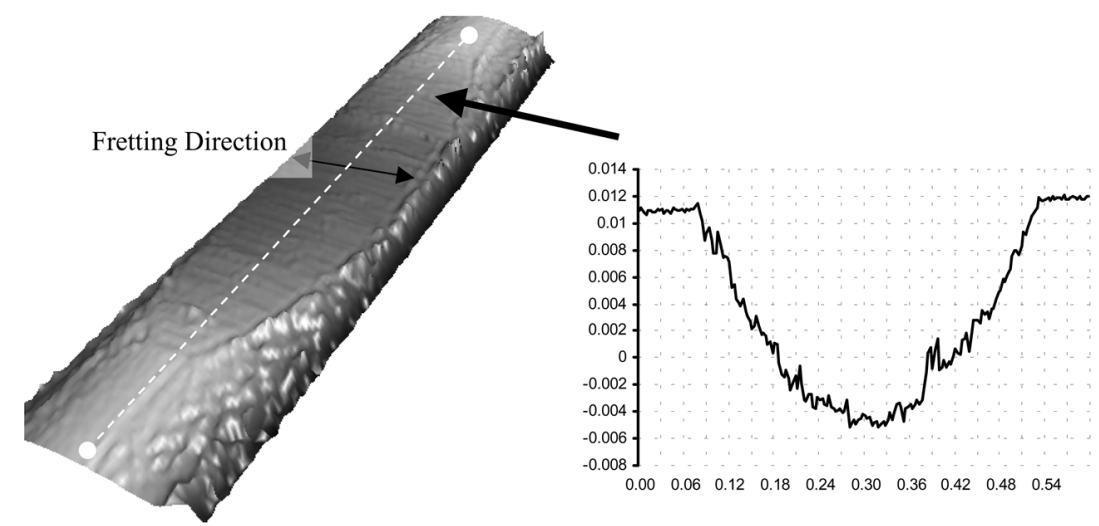

Fig. 6. (Left) Surface of wear of rider, $201 \times 51$ data, $0.6 \mathrm{~mm} \times 0.15 \mathrm{~mm}$, with (right) 2-D cross section along the length of the rider, with circle fit $(r=$ $1.627 \mathrm{~mm})$.

TABLE I

WeAr Data For All COUPON SURFACES

\begin{tabular}{|l|l|l|l|l|}
\hline Contact Force Level (N) & 1.75 & 0.75 & 0.15 & 0.05 \\
\hline Overall length of wear track. (Fretting Direction) & $260 \mu \mathrm{m}$ & $230 \mu \mathrm{m}$ & $125 \mu \mathrm{m}$ & $125 \mu \mathrm{m}$ \\
\hline Inner wear track length (Fretting Direction) & $100 \mu \mathrm{m}$ & $100 \mu \mathrm{m}$ & $100 \mu \mathrm{m}$ & $100 \mu \mathrm{m}$ \\
\hline Length of Wear Area A & $450 \mu \mathrm{m}$ & $120 \mu \mathrm{m}$ & $65 \mu \mathrm{m}$ & $65 \mu \mathrm{m}$ \\
\hline Maximum wear depth below datum & $10 \mu \mathrm{m}$ & $0.7 \mu \mathrm{m}$ & $0.5 \mu \mathrm{m}$ & -- \\
\hline Maximum wear height above datum & $9 \mu \mathrm{m}$ & $1.6 \mu \mathrm{m}$ & $0.8 \mu \mathrm{m}$ & - \\
\hline Volume below datum $\times 10^{-5} \mathrm{~mm}^{3}$ & 8.8 & 2.0 & 0.35 & -- \\
\hline Volume above datum $\times 10^{-5} \mathrm{~mm}^{3}$ & 9.8 & 1.5 & 0.03 & -- \\
\hline
\end{tabular}

TABLE II

WEAR DATA FOR CONTACT FORCE OF $1.75 \mathrm{~N}$ AND THE SURFACE SHOWN IN FIG. 6

\begin{tabular}{|l|l|}
\hline Overall length of wear, along the wire. & $450 \mu \mathrm{m}$ \\
\hline Inner wear track length & $\mathrm{N} / \mathrm{A}$ \\
\hline Maximum wear depth & $15.4 \mu \mathrm{m}$ \\
\hline Maximum wear height & $\mathrm{N} / \mathrm{A}$ \\
\hline Volume below datum $\times 10^{-5}$ & 31.5 \\
\hline Volume above datum $\times 10^{-5}$ & 1.2 \\
\hline
\end{tabular}

becomes more erratic, as a result of the increased peaks in the maximum resistance.

Fig. 8 shows a build-up of material towards the end of the wear track, and the width of the track (A) is much reduced over the $1750 \mathrm{mN}$ samples, where $450 \mu \mathrm{m}$ is reduced to $120 \mu \mathrm{m}$, in Table I. The volumetric loss from the surface, shown as the volume below the datum is much reduced when compared to the $1.75 \mathrm{~N}$ data. There is little damage or surface wear apparent on the rider surface, thus wear parameters are not defined. The same holds true for the rider surfaces, at 0.15 , and $0.05 \mathrm{~N}$.

\section{Intermittency and Surface Wear At Contact Force of $150 \mathrm{mN}$}

The contact resistance characteristic during the intermittency events are shown in Fig. 9 for 100 and 2500 cycles. This shows the mean resistance increasing over the first few cycles to a peak of $0.9 \Omega$, then reducing to a stable value of $0.4 \Omega$, at 40 cycles before raising again to a $0.8 \Omega$ peak. After 500 cycles the mean resistance becomes stable to 2500 cycles at approximately $0.25 \Omega$.

The wear track in Fig. 10 shows a much reduced wear profile when compared to the previous studies, which is reflected in the magnitude of the wear shown in Table II. It should be noted here that at this level of wear the volumetric parameters registered in Table I, are consistent with the values for the volumetric parameters of the unworn surface. The resulting wear track appears as a small change in the surface form. The 2-D cross section of the wear shows a small build-up of material toward the end of the track, with a smoothing of the asperities along the track. There is no apparent wear on the rider surface.

\section{Intermittency and Surface Wear At Contact Force of $50 \mathrm{mN}$}

The mean and minimum contact resistance characteristic during the intermittency events are shown in Fig. 11 over 2500 cycles. This shows the mean resistance increasing over the first 20-30 cycles to a peak of $1.1 \Omega$, then reducing to a value of $0.7 \Omega$. With no intermittencies recorded between 200-300 cycles no resistance values are presented. 

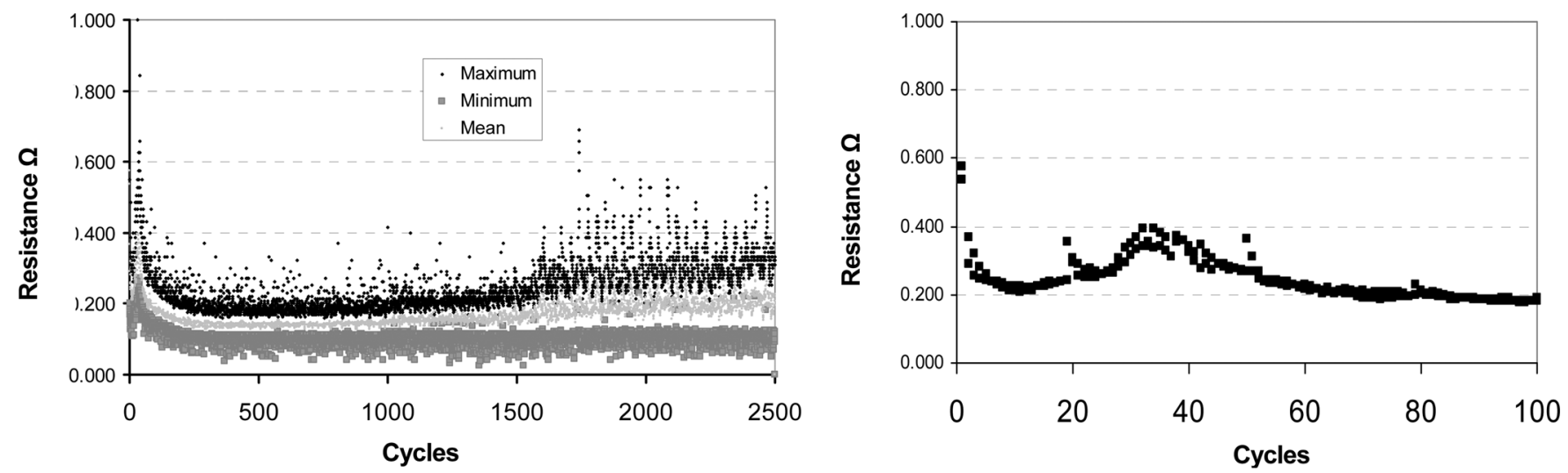

Fig. 7. Mean, Max and Min contact resistance over 2500 and 100 cycles during intermittency events at a constant contact force of 750 mN.
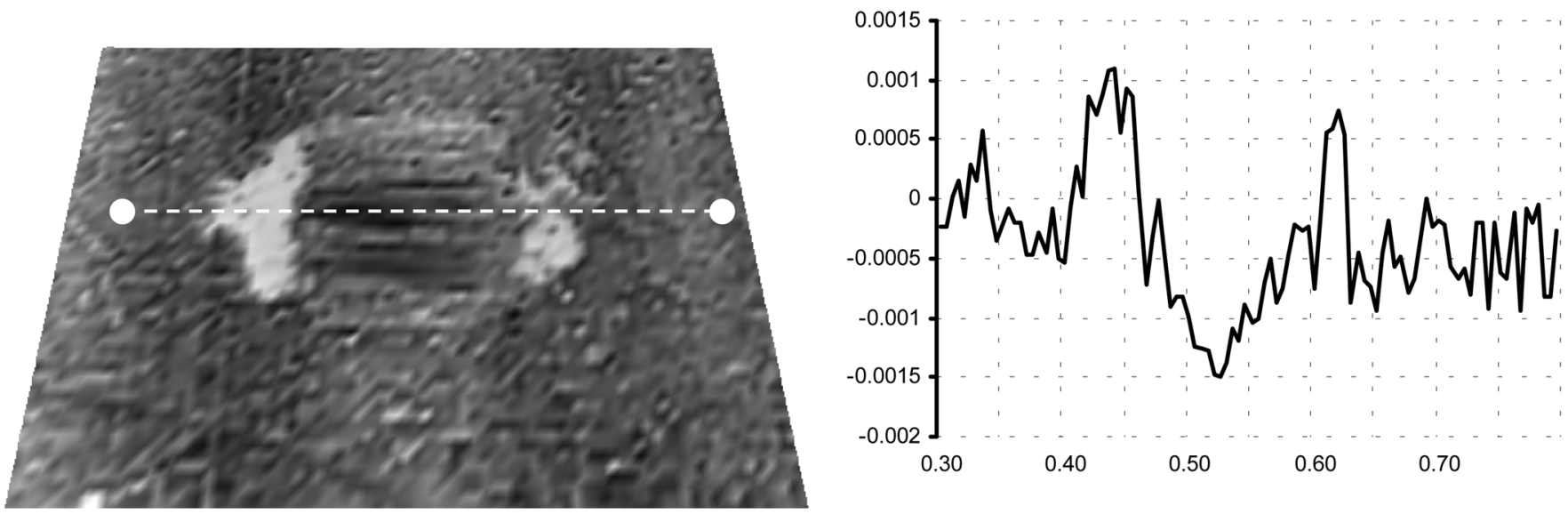

Fig. 8. Surface wear of coupon with the cylinder form is removed. (left) Showing the fretting direction of the rider, A the length of the wear area (right) and the 2-D cross section, all dimensions ( $\mathrm{mm}$ ).

\section{E. Wear At Contact Force of $50 \mathrm{mN}$}

At a contact force of $50 \mathrm{mN}$ there is no apparent wear on either the rider or the coupon surface. The only representation that can be achieved is by using the intensity of the reflected light, as shown in Fig. 12 where the reflectivity is increased as a result of a smoothing of the surface roughness. It is not possible to determine wear values for the surfaces. The resistance data shows a surface where it can be expected that most of the data correspond to oxide conduction.

\section{DISCUSSION}

\section{A. Relationship Between Intermittency, Wear and Contact Force}

Figs. 4, 7, 9, and 11 show the progression of the contact resistance during the intermittency events with contact force. The response at $1.75 \mathrm{~N}$ in Fig. 4 shows two peaks in the mean resistance, one at 50 cyles and one at 750 cycles. The first peak has been identified as the onset of the surface wear after the oxide has been removed. The initial metallic interaction of the surfaces is exhibitted by the lack of intermittent events around 20 cycles. This is followed by a period of low resistance and a second phase of mettalic contact around 500 cycles. This is followed by the second peak in the resistance. Between 1300 and
2500 cyles there is stability in the monitored resistance. This is expeceted to be related to a gross wear process where material is continuously removed. The final minimum resistance value at 2500 cycles is related to the metallic conduction phase. It is apparent that the selected surface exhibits intermittency over most of the 2500 cycles after an initial settling period. With the wear process related to the length of the fretting cycle (x) as stated in (2), a reduction in $x$ could be expected to have the same influence as a reduction in the applied force.

The resistance response in Fig. $7(0.75 \mathrm{~N})$ shows two peaks over the first 100 cycles, comparred to the one peak in Fig. 4 $(1.75 \mathrm{~N})$. The lower force will reduce the wear and removal of oxides, thus intermittency events occur during every cycle of the first 100 . The increase in the mean resistance around 1500 cyles is thought to be related to the second increase in Fig. 4, around 700 cycles.

The data in Fig. $9(0.15 \mathrm{~N})$ has a similar characteristic to that in Fig. $7(0.75 \mathrm{~N})$, with the peaks occuring at a later stage. The data in Fig. $11(0.05 \mathrm{~N})$ shows intermittency events during most of the initial 100 cycles, with a period around 100 cycles where metallic conduction occurs.

The study shows that the contact force is related to the fretting cycle at which the phase of the wear interaction occurs. It is proposed that the length of the fretting cycle (x) will have a similar influence. 

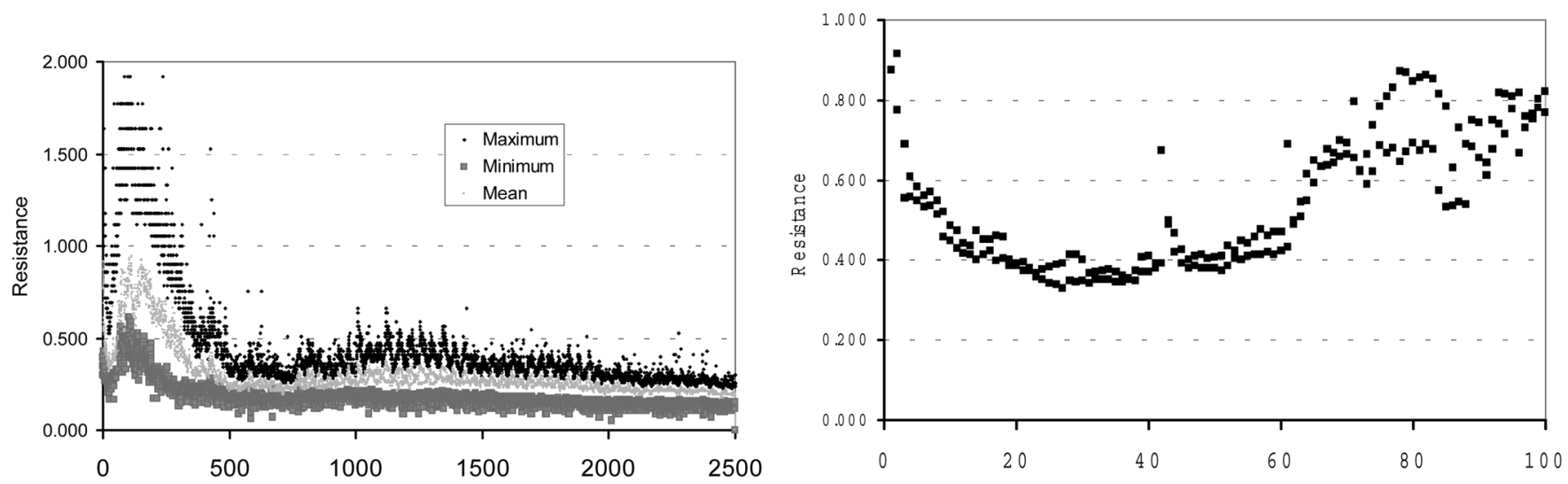

Fig. 9. Mean, Max and Min contact resistance over 2500 and 100 cycles during intermittency events at a constant contact force of $150 \mathrm{mN}$.
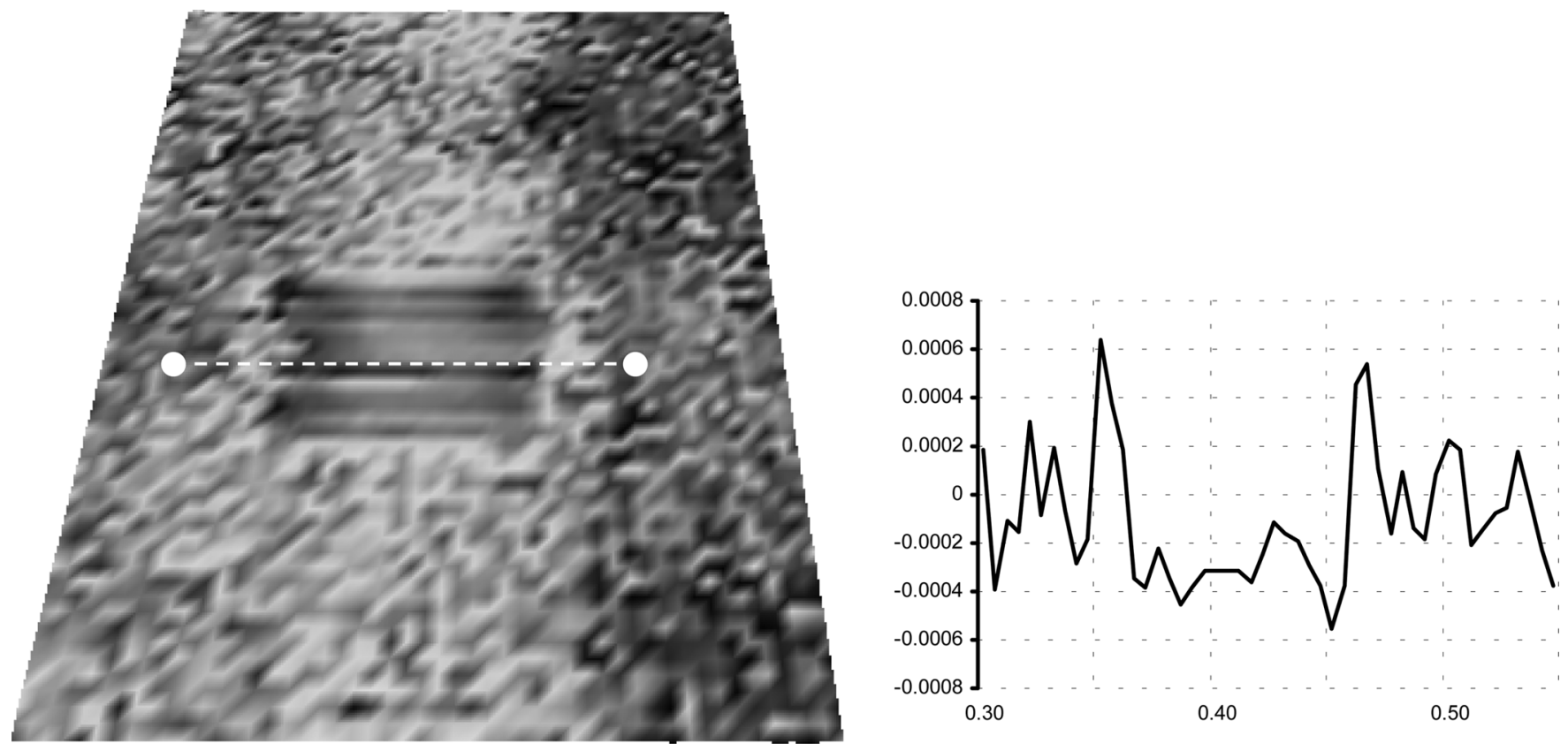

Fig. 10. Surface wear of coupon with the cylinder form is removed. (left) Showing the fretting direction of the rider, A the length of the wear area and the (right) 2-D cross section, all dimensions $(\mathrm{mm})$.
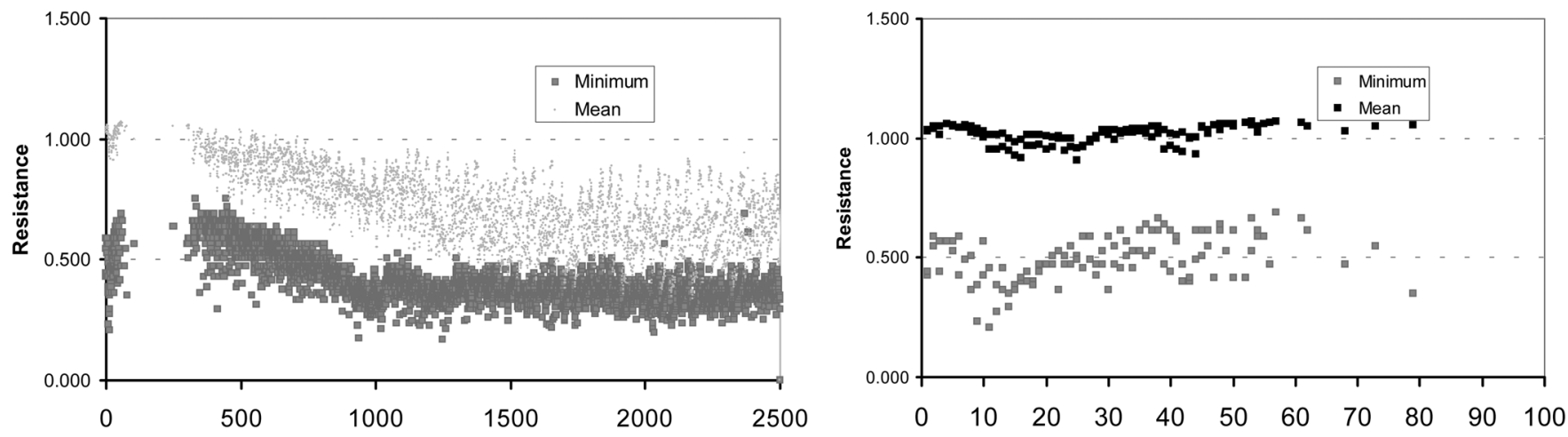

Fig. 11. Mean, max, and min contact resistance over 2500 and 100 cycles during intermittency events at a constant contact force of $50 \mathrm{mN}$. 


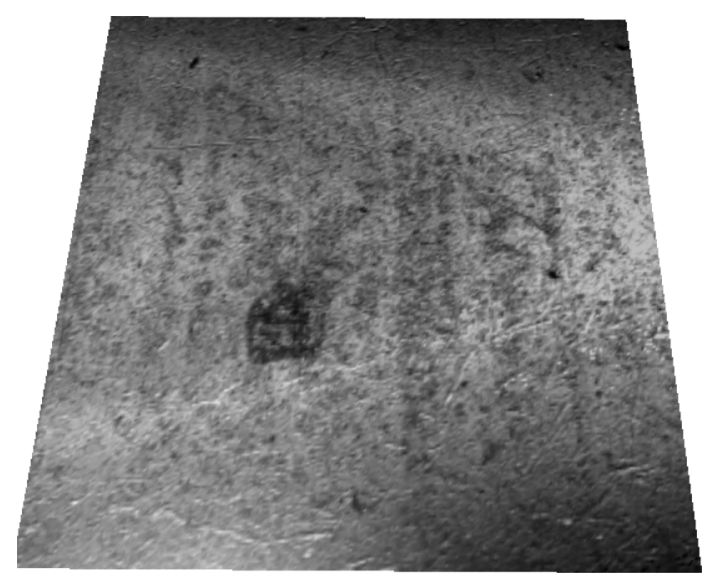

Fig. 12. Reflected light intensity, $251 \times 251$ data, $1.25 \mathrm{~mm} \times 1.25 \mathrm{~mm}$.

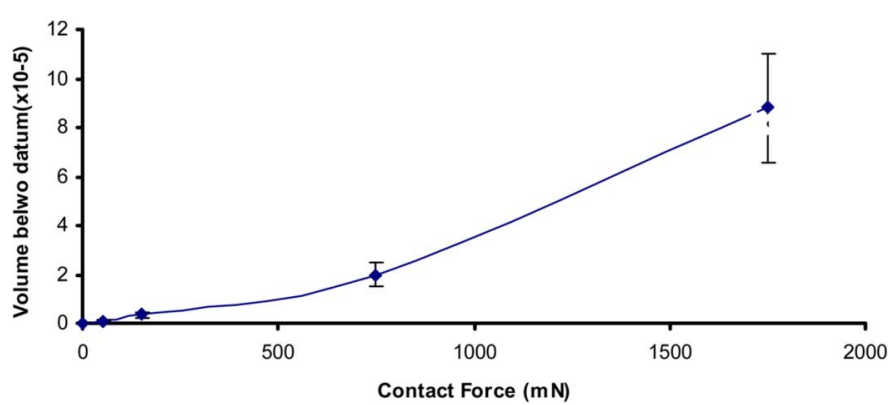

Fig. 13. Volumetric wear of the coupon surface, based on the data in Table I.

\section{B. Wear Study}

To investigate the wear process the amount of material removed from the coupon surface is investigated. With limited samples and with uncertainty associated with the volumetric data, the trend in Fig. 13 is presented as an indication of the expected increase in wear with contact force, in line with the model expressed in (2). There are a number of potential error sources associated with the volumetric wear calculation; the most significant is the relationship to the surface volumes for the unworn surface over the same area as that used for the calculation in Table I. It has been estimated that for the surfaces used in this study, that the volume below the datum for an unworn surface, which is related to the surface roughness is approximately $25 \%$ of the volume fraction for a given area. This has been accounted for in the errors bars in the Fig. 13.

\section{Relationship Between Contact Force and Contact Resistance}

With the continuous monitoring of the contact volt-drop and thus the contact resistance, and the variation in data over the 2500 cycles, it is necessary to determine a single value for use in a modeling process. For this purpose it is proposed that the minimum resistance after the 2500 cycles is used [9]. This value generally corresponds to a period of stability after the initial wear periods characterized by the two peaks in Fig. 4. At 2500 cycles the resistance will be composed of a mixed conduction process of metallic the lower value and through oxides the maximum value. The results are shown in Fig. 14, and are compared to the theoretical values based on the standard representation of

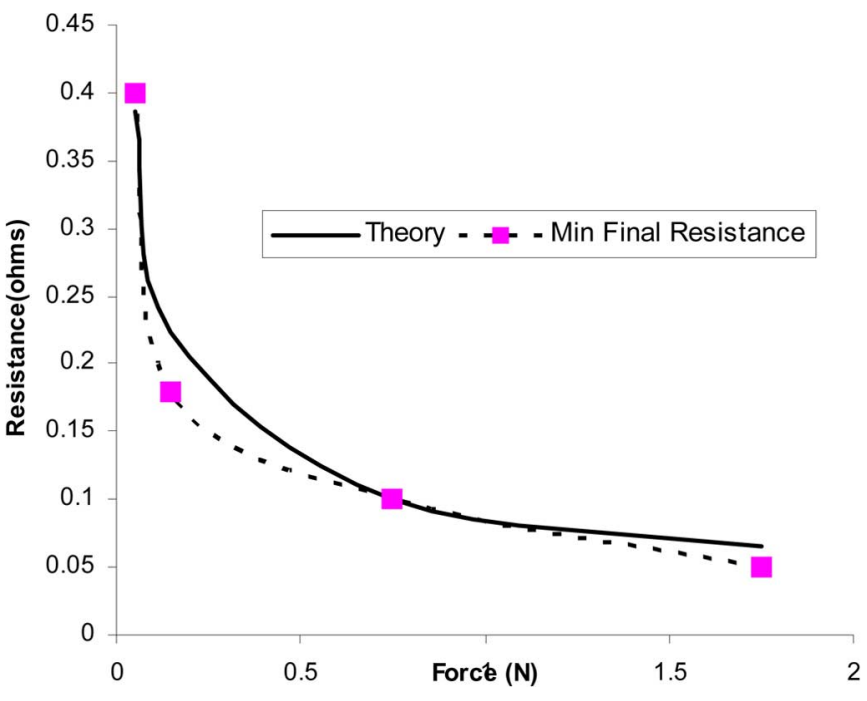

Fig. 14. Min final resistance after 2500 cycles, with the applied force, compared to the predicted resistance based on (3) with $\eta=2.7$.

resistance as a function of contact force, shown in (3). The equation is based on a number of assumptions, [10], and is used here with $\eta=2.7$, implying some surface oxide interaction. The maximum resistance will be linked to the conduction process through surface oxides and other debris.

The data shows that the minimum resistance value provides a satisfactory mapping to the experimental data, as there are a number of approximations in the estimation of the material parameters. The maximum resistance values are not used as the interaction with the oxide film towards the ends of the track provides inconsistent data

$$
R_{c}=\left[\frac{\rho^{2} \eta \pi H}{4 F}\right]^{1 / 2}
$$

where:

$$
\begin{array}{ll}
F & \text { force }(\mathrm{N}) \\
\rho & \text { resistivity }\left(1.033 \times 10^{-6} \Omega \mathrm{m}\right) \\
\eta & 2.7 ;(\eta=1 \text { for an oxide free surface); } \\
H & \text { hardness }\left(3 \times \sigma_{y}\right) ; \text { where } \sigma_{y}=414 \times 10^{6} \mathrm{~N} / \mathrm{m}^{2} .
\end{array}
$$

\section{Performance Opimization}

In the design of connector systems where fretting is a feature of the degradation mechanism, a higher contact force will provide a lower resistance, but at the cost of surface wear (Fig. 13). At the lower contact force levels the oxide film does not appear to be degraded leading to high variation in the contact resistance during a single cycle. An optimum force level is an intermediate force, with reduced fretting amplitude. It is important to note that in real connector systems the contact force is not maintained, but could be expected to fall with the wear process, leading to a lowering of the applied force and an associated fall in the wear rate. For the solid material selected for this study the optimum performance appears to be at the higher force levels, where there is always some metallic conduction in the middle of the fretting cycle. 


\section{E. Intermittency}

As the connector surface wears, the intermittency events will continue as at all stages there is interaction between oxide films. The model for the wear process has been shown to hold for the crossed-rod configuration, for the higher force levels, but with the addition of a high number of events during the first 10 cycles.

The model does not hold at low force levels where there is little apparent wear of the surface. The number of intermittency events shows that as the contact force is reduced there is an increasing likelihood of conduction through the oxide films. At the lowest force levels tested here, the material shows that dynamic resistance is dominated by oxide conduction processes.

\section{CONCLUSION}

The results show the potential difficulties in using electrical connecting system in in-vivo applications, where the selected surfaces are subject to oxide or other contamination. The amplitude of the fretting cycle will have a critical influence on the surface performance, and the relationship to intermittency events. With fretting apparent on a surface the results show that intermittency events occur at all stages, and at all force levels, when using a $20 \mathrm{mV}$ supply.

The application of the crossed-rods test configuration, results in a complex wear process which enhances the intermittency events. This occurs as both the form of the rider and the coupon surface are changed, by the wear process. The model previously proposed for the interaction between the wear process and intermittency events has been confirmed for the crossed-rod configuration, but has been extended to include a fourth stage at higher force levels, where the surfaces exhibit a high level of wear on both rider and coupon.

The minimum of the dynamic resistance during a single $1 / 2$ cycle has been shown to be the parameter linked to established contact theory, with a factor of 2.7 to account for the surface films.

\section{ACKNOWLEDGMENT}

The author would like to thank Dr. C. Maul, TaiCaan Technologies, Ltd., for his contribution to the experiment data collection.

\section{REFERENCES}

[1] J. W. McBride and C. Maul, "Intermittency events in bio-compatible electrical contacts," in Proc. 51st IEEE Holm Conf. Elect. Contacts, 2005, pp. 75-81.

[2] C. Maul, J. W. McBride, and J. Swingler, "Intermittency phenomena in electrical contacts," IEEE Trans. Compon. Packag. Technol., vol. 24, no. 3, pp. 370-377, Sep. 2001.

[3] W. Abbott and K. Schreiber, "Dynamic contact resistance of gold tin and palladium connector interfaces during low amplitude motion," in Proc. Holm Conf. Elect. Contacts, 1981, pp. 211-219.

[4] C. Maul and J. W. McBride, "A model to describe intermittency phenomena in electrical contacts," in Proc. IEEE Holm Conf. Elect. Contacts, 2002, pp. 165-174.

[5] S. R. Murrell and S. L. McCarthy, "Intermittence detection in fretting corrosion studies of electrical contacts," in Proc. 43rd Holm Conf. Elect. Contacts., 1997, pp. 1-7.

[6] Standard Test Methods for the Evaluating Connectability Characteristics of Electrical Conductor Materials, ASTM B 896-99, 2005.

[7] J. W. McBride, A. P. Sumption, and J. Swingler, "On the evaluation of low level contact erosion," in Proc. 50th IEEE Holm Conf. Elect. Contacts 22nd Int. Conf. Elect. Contacts, Sep. 20-23, 2004, pp. 370-377.

[8] Tai Caan Technologies, http://www.taicaan.com, 2008.

[9] J. W. McBride, "A review of volumetric erosion studies in low voltage electrical contacts," IEICE Trans. Electron., vol. E86-C, no. 6, pp. 908-914, 2003.

[10] Electrical Contacts: Priciples and Applications, P. Slade, Ed. New York: Marcel Dekker Inc, 1999, pp. 12-12.

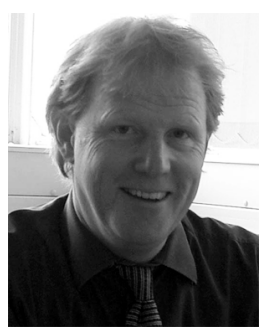

John W. McBride is a Professor of electro-mechanical engineering in the School of Engineering Sciences, University of Southampton, Southampton, U.K. He is Chair of the Electro-Mechanical Research Group, and was previously Head of Research in the School (2001-2005). His main research interests include electrical contacts, metrology, and instrumentation.

Dr. McBride received the IEEE Holm Scientific Achievement award in 2006. He is an Associate Editor of the IEEE TRANSACTIONS ON COMPONENTS AND PACKAGING TEChNOLOGIES and a member of the Organizing Committee, IEEE Holm Conference on Electrical Contacts. 